\title{
Differential signaling of Flt3 activating mutations in acute myeloid leukemia: a working model
}

\author{
Perry M. Chan ${ }^{凶}$ \\ STAR, Singapore 138673, Singapore \\ $\triangle$ Correspondence: Perry.Chan@nrp.a-star.edu.sg \\ Received January 28, 2011 Accepted February 7, 2011
}

Neuroscience Research Partnership, Biomedical Sciences Institute, affiliated with Institute of Molecular and Cell Biology, A-

\begin{abstract}
Receptor tyrosine kinases couple a wide variety of extracellular cues to cellular responses. The class III subfamily comprises the platelet-derived growth factor receptor, c-Kit, Flt3 and c-Fms, all of which relay cell proliferation signals upon ligand binding. Accordingly, mutations in these proteins that confer ligand-independent activation are found in a subset of cancers. These mutations cluster in the juxtamembrane (JM) and catalytic tyrosine kinase domain (TKD) regions. In the case of acute myeloid leukemia (AML), the juxtamembrane (named ITD for internal tandem duplication) and TKD Flt3 mutants differ in their spectra of clinical outcomes. Although the mechanism of aberrant activation has been largely elucidated by biochemical and structural analyses of mutant kinases, the differences in disease presentation cannot be attributed to a change in substrate specificity or signaling strength of the catalytic domain. This review discusses the latest literature and presents a working model of differential FIt3 signaling based on mis-localized juxtamembrane autophosphorylation, to account for the disease variation. This will have bearing on therapeutic approaches in a complex disease such as AML, for which no efficacious drug yet exists.
\end{abstract}

KEYWORDS acute myeloid leukemia, receptor tyrosine kinase, oncogenic mutation, autoinhibition, intracellular trafficking

\section{INTRODUCTION}

Acute myeloid leukemia (AML) is characterized by a rapid expansion of myeloid white blood cancer cells, which compete against and block the function of normal bone marrow cells. The standard treatment regimen is conventional chemotherapy combined with, depending on the subtype diagnosis, hematopoietic stem cell transplantation. As the most common acute leukemia in adults, AML is extensively studied for the genetic and biochemical underlying causes, in the hope of developing more efficacious and targeted therapies. Flt3, a receptor tyrosine kinase normally signaling the proliferation of hematopoietic stem and progenitor cell in development (Matthews et al., 1991; Small et al., 1994), is mutated in a significant proportion $(25 \%-30 \%)$ of AML patients (Nakao et al., 1996; Yamamoto et al., 2001; Stirewalt and Radich, 2003; Meshinchi and Appelbaum, 2009). As the most established molecular marker in AML, Flt3 is a well-studied kinase for its activation and downstream signaling pathways (Meshinchi and Appelbaum, 2009; Masson and Rönnstrand, 2009). Perhaps the most puzzling observation is that although mutations in different regions of Flt3 lead to the same biochemical result of constitutive kinase catalytic activity, these mutations markedly segregate in their correlation to disease representation and prognosis. This review covers recent studies that attempt to provide a mechanistic basis for this segregation and offers a working model in line with the evidence, in the hope of revealing novel avenues for therapy.

\section{STRUCTURE AND FUNCTION OF FLT3}

Flt3 (Fms-like tyrosine kinase 3) is a member of the transmembrane receptor tyrosine kinase superfamily. It is grouped together with PDGFR (platelet-derived growth factor receptor), Fms and c-Kit within the type III tyrosine kinase domain (TKD) family (Gilliland and Griffin, 2002), based on the common characteristics of an immunoglobulin-like extracellular ligand-binding domain, a transmembrane domain, 
and an intracellular kinase domain with the two catalytic lobes interspersed by a relatively long kinase insert. Flt3 undergoes extensive glycosylation in the Golgi and the immature and mature forms run as $130-160 \mathrm{kDa}$ bands in gel electrophoresis. Upon binding of extracellular domains to the dimeric Flt3 ligand, the intracellular kinase domains position themselves for autophosphorylation in trans. This creates docking sites for phosphotyrosine binding domains in proteins involved in proliferative signaling, such as phospholipase $\mathrm{Cy}, \mathrm{SHC}$, GRB2, SRC (Dosil et al., 1993), SHP2 and GAB2 (Zhang and Broxmeyer, 1999), which converge downstream to activate AKT and ERK (extracellular signal-regulated kinase) mediated pro-survival and proliferative pathways. Targeted disruption of Flt3 in mice resulted in reduced populations of $B$ lymphoid progenitor cells and defective reconstitutive activity of transplanted stem cells in re-populating both lymphoid and myeloid cells (Mackarehtschian et al., 1995).

\section{AUTOINHIBITION OF FLT3 KINASE ACTIVITY}

Structural studies of Flt3, c-Kit and c-Fms kinase domains have shown the juxtamembrane (JM) region of type III RTKs to have an autoinhibitory role via intramolecular interactions with the catalytic domain (Griffith et al., 2004; Mol et al., 2004; Walter et al., 2007). JM interactions span the $\mathrm{N}$ and $\mathrm{C}$ terminal lobes of the catalytic domain to stabilize the autoinhibited conformation of the kinase, in which the $\mathrm{aC}$ helix is oriented away from coordinating the ATP binding motif to the catalytic Asp residue. Ligand-induced activation of wild-type receptor involves phosphorylation of conserved Tyr residues in the JM region, which is the earliest autophosphorylation event in the course of kinase activation, to disrupt these inhibitory contacts (Mol et al., 2003; Heiss et al., 2006). Oncogenic mutations in the JM region, however, bypass this requirement of trans autophosphorylation for alleviation of repression, leading to dramatically faster activation kinetics of the purified c-Kit catalytic domain in vitro (Chan et al., 2003) and of the full-length Flt3 receptor in cells (Razumovskaya et al., 2009). As type III RTK JM sequences are highly conserved (Fig. 1), this oncogenic mode of kinase activation is likely to apply for all the members of this subfamily. As the catalytic domain is the ultimate target of such repression, it is not surprising that

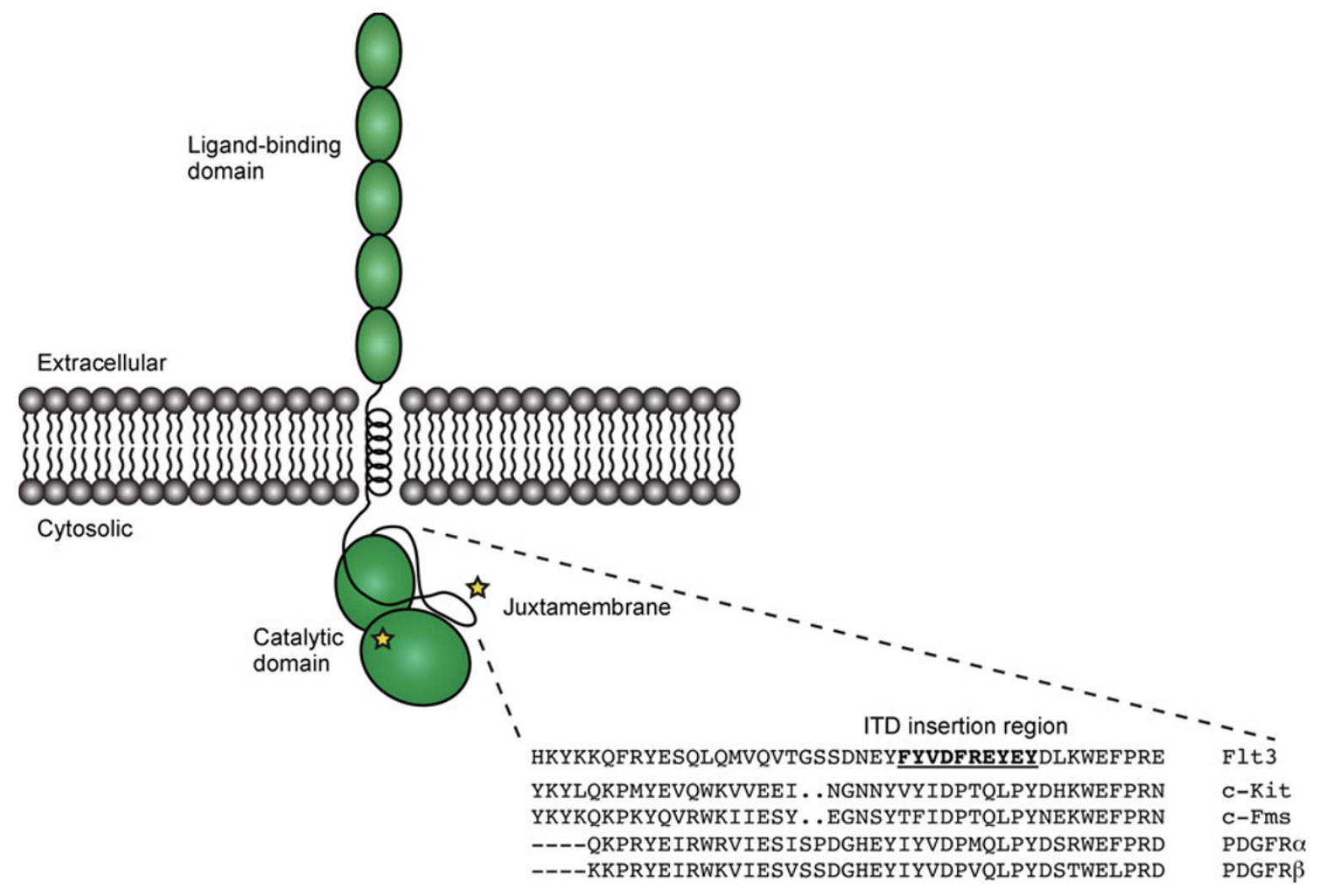

Figure 1. Schematic diagram of type III receptor tyrosine kinases (RTKs). Domain structures consist of the ligand-binding domain (five extracellular immunoglobulin-like folds represented by five green ovals), a single transmembrane pass, the intracellular juxtamembrane (JM) region (loop encompassing the catalytic domain), and the catalytic domain with N- and C-terminal lobes (represented by two green ovals). Stars represent the JM and activation loop mutational sites observed in AML. Shown below is the alignment for the full JM sequence of all five type III RTKs. The region in FIt3 JM with a high frequency of internal tandem duplication (ITD) insertions is in bold underlined font. 
mutations within the activation loop (i.e. Asp835) can directly stabilize the active conformation and similarly result in ligandindependent receptor signaling (Yamamoto et al., 2001).

\section{DIFFERENCES IN ONCOGENIC FLT3 SIGNALING}

If constitutive autophosphorylation alone is the definitive event in Flt3-causative AML, then mutations in the JM and catalytic domain that abolish autoinhibition should be equivalent in clinical presentations. However, this is not the case. JM mutations, which typically manifest themselves as internal tandem duplications (ITDs), are strongly associated with poorer prognosis and shorter progression-free survival compared to the activation loop (AL) mutations, particularly in the context of Flt3 WT loss of heterozygosity (Kiyoi et al., 1999; Kottaridis et al., 2001; Yamamoto et al., 2001; Meshinchi et al., 2006). It is possible that ITD and AL mutations originate differentially in myeloid cell subtypes, with ITD mutations favored in subtypes with greater oncogenic potential, but there is no clear study on this. In contrast, arguing against this scenario are studies that express ITD and AL mutations in the same cell type and yet more aggressive transforming properties are observed for the ITD mutant (Choudhary et al., 2005; Grundler et al., 2005). Clear differences in activation of downstream signaling pathways (Choudhary et al., 2005; Schmidt-Arras et al., 2009) and in transcription profiles (Tickenbrock et al., 2005; Lu et al., 2007) between the two classes of mutations have been reported. A switch in substrate specificity can account for these observations. Mutations in the kinase domain can lead to a switch in target preference, as in the case of c-Abl (Till et al., 1999). However, this is unlikely for FIt3 AL mutations because the studies cited above reported no significant deviation from Flt3 WT signaling pathways. The target preference switch may also arise from gain or loss of ITD recruitment of FIt3 kinase targets. STAT5 (signal transducer and activator of transcription 5) is activated via FIt3 ITD but not Flt3 WT or AL mutant signaling (Hayakawa et al., 2000; Choudhary et al., 2005) through an as yet unknown mechanism. This targeting mechanism is used by c-Abl in which its $\mathrm{SH} 2$ domain recruits substrates for phosphorylation by the adjacent catalytic domain (Mayer et al., 1995). There are conflicting reports on whether determinants in the Flt3 JM recruit Stat5, the best characterized downstream target of FIt3 ITD mutants (Kiyoi et al., 2002; Murata et al., 2003; Rocnik et al., 2006). To date there is no evidence of direct interaction between Stat5 and FIt3 JM. Also lacking in the literature is a direct comparison of recombinant intracellular FIt3 WT and ITD kinases on phosphorylation efficiency of full-length protein substrates. The in vitro comparison of the related c-Kit WT and JM mutant kinases showed no differences in substrate preference towards synthetic peptides (Chan et al., 2003), which in this context is inconclusive to support or rule out the recruitment mechanism. Another possible scenario involves Stat5 spe- cific activation via JM recruitment of tyrosine kinases and phosphatases (Heiss et al., 2006). This alternative recruitment scheme apparently has also been difficult to demonstrate, with one report ruling out the involvement of Src family or JAK kinases in Stat5 activation (Choudhary et al., 2007). Thus, differences in the clinical outcomes between ITD and AL mutation associated AML patients cannot be satisfactorily explained so far by differences of cell origin or a switch in substrate recognition. The differential frequencies of ITD and AL mutations associated with AML, $25 \%$ for ITD and $7 \%$ for $A L$, further highlight their differences (Gilliland and Griffin, 2002; Levis and Small, 2003). To account for these observations, a working model of FIt3 ITD versus AL signaling is presented as follows.

\section{A WORKING MODEL OF DIFFERENTIAL SIGNALING}

The autoinhibited and active kinase structures of the related c-Kit kinase provide insight into FIt3 ITD activation (Mol et al., 2004). The authors concluded that the length of the JM region, which is also conserved among all type III RTKs (Fig. 1), precludes autophosphorylation in cis. The structure of activated c-Kit showed that JM autophosphorylation sites Tyr568/570 are about 25 angstroms away from the catalytic Asp residue of the same molecule, which is too far for phosphotransfer. In the structure of autoinhibited Flt3 kinase, the JM autophosphorylation sites Tyr589/591 are $\sim 15$ angstroms away from the catalytic Asp (Griffith et al., 2004), ruling out intramolecular phosphotransfer (Fig. 2A). These sites are required for FIt3 ITD mediated phosphorylation of Stat 5 and efficient myeloproliferation of bone marrow transplanted cells (Rocnik et al., 2006). Therefore it seems that the JM maintains its repressive function by fulfilling two requirements: (1) intramolecular interactions with the kinase domain and (2) inaccessibility to the active site of the same molecule. FIt3 ITD insertions range widely in size-one survey reported 15-153 nucleotides with the median length of 39 bases (Stirewalt et al., 2006). The minimal FIt ITD insertion of 15 bases would add 5 amino acids, which in an extended conformation lengthens the JM by about 15 angstroms. Therefore even the minimal ITD mutation is sufficient to bridge Tyr589/591 sites to the Asp in the catalytic loop, leading to autophosphorylation in cis (Fig. 2B). Significantly, the subgroup of patients with ITDs smaller than the median length had a doubled survival rate $(43 \%)$ than the subgroup with ITDs larger than the median $(21 \%)$ over a 5 -year period. ITD insertions usually fall within the span of amino acids 590-600 (Fig. 1) (Levis and Small, 2003). The relationship of ITD length and prognosis will be discussed later.

Another relevant point is the difference in intracellular trafficking patterns. Exogenously expressed FIt3 WT and ITD proteins have been reported with the WT protein localized predominantly at the plasma membrane and ITD mutant 
retained at the perinuclear region (Koch et al., 2008). This would result in differential signaling because FIt3 ITD targeted to the plasma membrane via myristoylation showed significantly reduced Stat5 activation concomitant with increased Erk and Akt phosphorylation (Choudhary et al., 2009). Therefore it appears that the ITD mutation acts as a switch for aberrant trafficking of the receptor kinase, permitting access to substrates not localized at the plasma membrane.

Taking these data together, a working model of FIt3 ITD and $\mathrm{AL}$ mutant signaling is illustrated in Fig. 2 and 3 . The altered JM of FIt3 ITD is uncoupled from the kinase domain, destabilizing the autoinhibited kinase conformation. As the nascent mutant protein is synthesized in the endoplasmic reticulum (ER), target sites of the lengthened JM including Tyr589/591 reach the catalytic cleft, resulting in highly efficient autophosphorylation (Fig. 2B). Longer ITD insertions allow greater degree of freedom for JM target sites to access the catalytic cleft in cis and result in a higher proportion of autophosphorylated JM FIt3 ITD. FIt3 ITD trafficking along the ER-Golgi network is retarded through interactions of the phospho-JM motifs with phosphotyrosine binding domain (PTD) containing proteins such that the majority of FIt3 ITD does not reach the plasma membrane (Fig. 3). Examples of interacting proteins include $\mathrm{c}-\mathrm{Cbl}$, which has been shown to bind c-Kit via interaction of its PTD and the phospho-c-Kit JM (Masson et al., 2006) and to directly bind FIt3 ITD constitutively although no site was mapped (Sargin et al., 2007). Other candidate interacting proteins would be ERand/or Golgi-resident proteins that recognize the Flt3 JM phospho-motifs. The prolonged cytosolic retention of FIt3 ITD increases exposure time to substrates such as Stat5. The JM of Flt3 AL mutant is likely also uncoupled from the kinase domain, but is inefficiently autophosphorylated as the nascent protein is synthesized. The normal length of the FIt $3 \mathrm{AL} \mathrm{JM}$ is insufficient for target sites to reach the catalytic cleft in cis, but requires another $\mathrm{AL}$ mutant kinase in close proximity for autophosphorylation in trans. This results in only a fraction of phosphorylated JM FIt3 AL mutant relative to FIt3 ITD to undergo the same processing delay as FIt3 ITD. This is supported by the report of Tyr589/591 also being required for ligand-independent proliferation of cells expressing Flt3 AL (Vempati et al., 2008). The non-phosphorylated JM Flt3 AL fraction is efficiently processed and traffics to the plasma membrane, and would transmit downstream signals similarly to those of Flt3 WT. In summary, FIt3 ITD presents a higher load (compared to FIt3 AL) of intracellular phospho-JM kinase arising from efficient autophosphorylation, and is more accessible to substrates not proximal to the plasma membrane. ITD length is proportional to JM autophosphorylation efficiency-this helps explain the lower survival rate in patients with longer ITDs. It is possible that the higher load of phospho-JM FIt3 ITD can result from autophosphorylation in trans also, as the lengthened JM can conceivably be more accessible to a proximal Flt3 kinase.

\section{CONCLUSION AND FUTURE PROSPECTS}

It has been 15 years since mutations in Fit3 were found to associate with leukemias (Nakao et al., 1996). Despite extensive studies of Flt3 oncogenic signaling in that time (Masson and Rönnstrand, 2009), fundamental questions remain unresolved. The observation that FIt3 ITD and $A L$ activating mutations have different clinical presentations despite having similar in vitro characteristics remains a conundrum in AML etiology. The collection from structural, cellular trafficking, animal model and patient genotyping studies suggests the scenario presented in Fig. 3, called the "intracellular active kinase load" model.

There remains much experimentation to prove this model. Biochemical assays measuring activation kinetics of recombinant Flt3 kinases can distinguish between cis and trans autophosphorylation events. The starting point of such assays must use dephosphorylated Flt3, which can be generated by treatment with protein tyrosine phosphatases (Chan et al., 2003). cis versus trans JM autophosphorylation can be distinguished by the activation lag times of titrated kinase concentrations- cis would result in concentrationindependent lag times and trans in concentration-dependent lag times. Flt3 $\mathrm{AL}$ and ITD of varying lengths can be characterized in this way. The intracellular trafficking patterns of Flt3 proteins can be tracked in live cells using a fusion photoactivatable tag. This can determine protein half-life, trafficking rate and route. Antibodies directed against Flt3 pTyr589/591 tandem sites that can be used in cryo-electron microscopy must be developed to definitively localize WT and mutant Flt3. Stat5 is the most established biomarker to distinguish FIt3 ITD and AL signaling, but it is not an attractive target therapeutically. To identify more suitable candidates, proteomic studies using SILAC (Stable Isotope Labeling with Amino acids in Cell culture) mass spectrometry (Ong et al., 2002; Zhu et al., 2002) should be performed to characterize Flt3 mutant differential downstream signaling. This approach can quantitate relative levels of tyrosine phosphorylated proteins in cell lines expressing FIt3 ITD and AL mutants.

Alternative models cannot be ruled out at this point because the current data is not conclusive. There are conceivably a plethora of alternate models that can replace or work in tandem with the working model presented here. First, there is the issue of ITD and AL mutations originating in specific stages of the myeloid lineage, i.e. pluripotent stem cell versus myeloid precursor. The mechanisms of generating tandem duplication and point mutations must be different, but it is unknown whether any specific cell stage is more prone to one over another. AML is one of the first cancers demonstrated to involve early lineage hematopoietic cells giving rise to the proliferative leukemic cells (Huntly and Gilliland, 2005). The current paradigm, although still strongly debated, is that the transformed stem cell is the lynchpin behind the cancer. If ITD mutations are favored in transformed cells with stem-like 
A

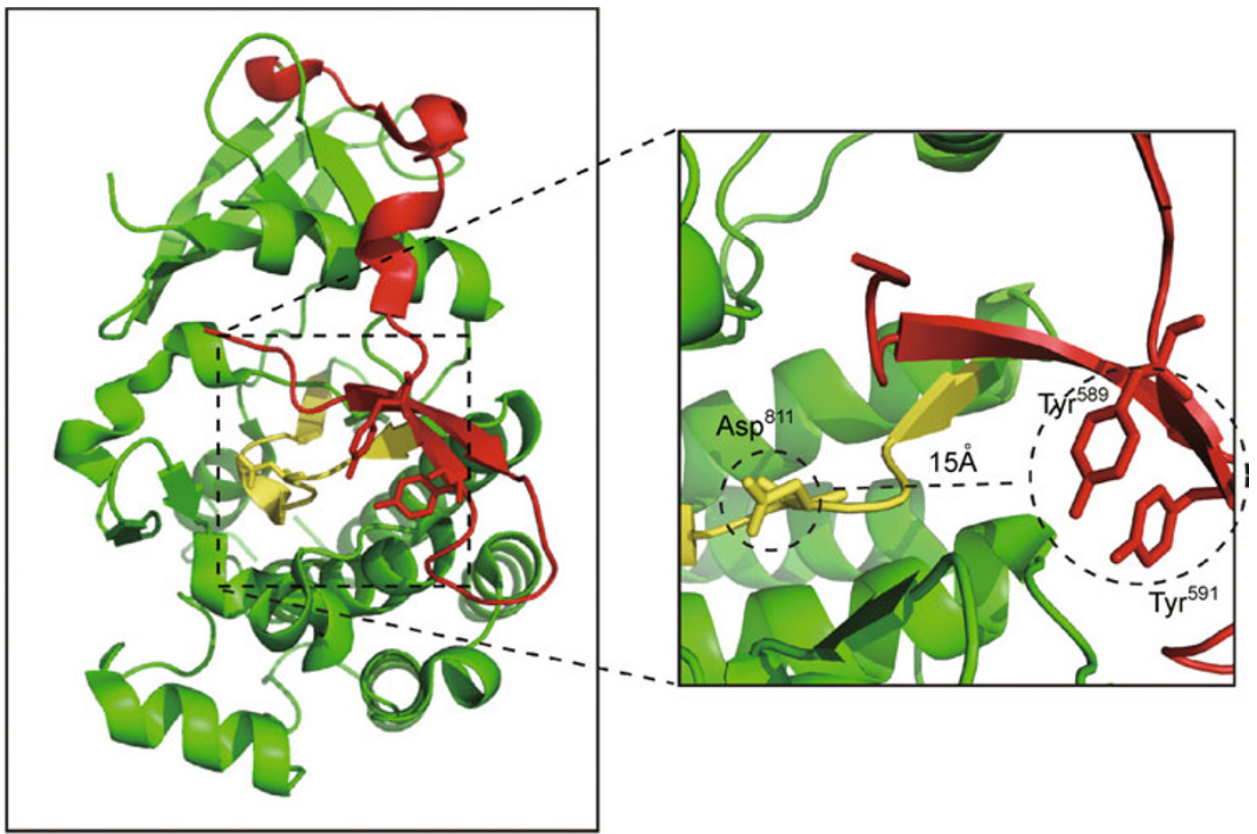

B

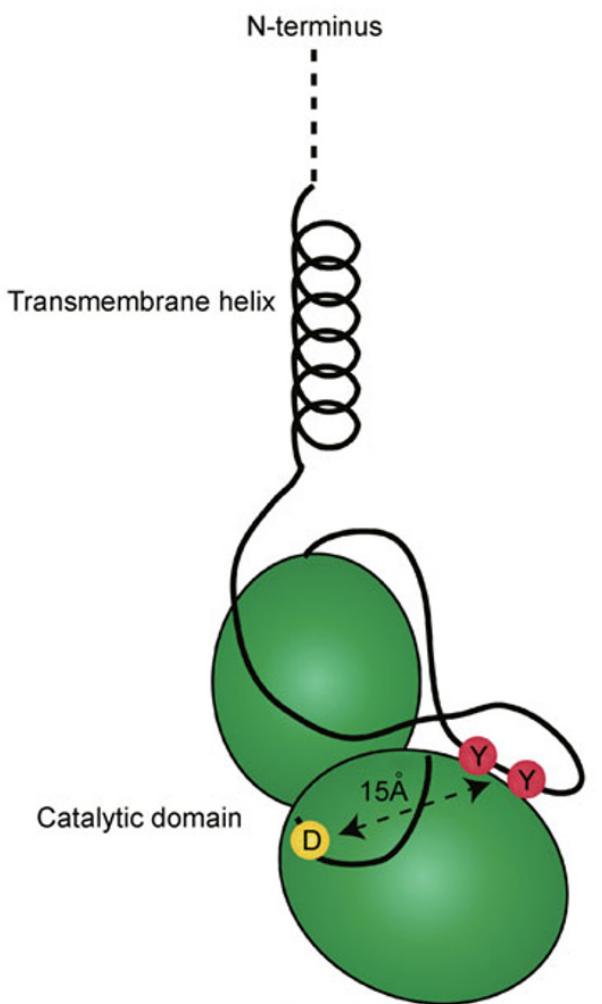

Wild-type, autoinhibited

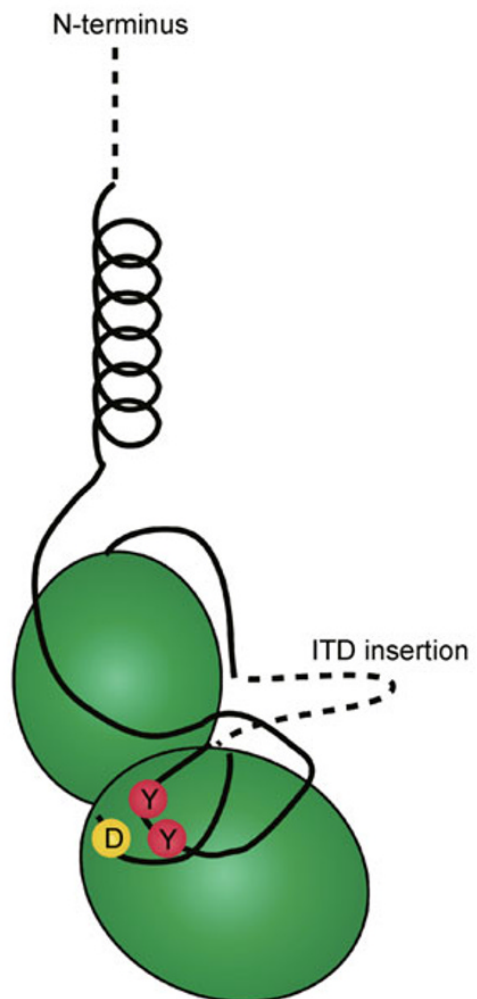

ITD mutant, activated

Figure 2. Autoinhibited FIt3 kinase structure and schematic diagram of autoinhibited FIt3 WT and activated FIt3 ITD. (A) Ribbon representation of autoinhibited Flt3 kinase structure (Griffith et al., 2004). The juxtamembrane region is colored in red and the catalytic loop in yellow. The inset shows the relative positions of catalytic Asp811 to the autophosphorylation sites Tyr589 and 591 (side chains drawn as sticks). The images were produced using PyMol. (B) A schematic diagram of autoinhibited Flt3 WT and activated FIt3 ITD. The two lobes of the catalytic domain are represented by green ovals, the JM region by a flexible line adjoining the $\mathrm{N}$-terminal lobe (spanning the two lobes in the wild-type autoinhibited conformation) and the activation loop by a semicircle near the intersection of the two lobes. The transmembrane span is represented by a helix, Tyr589/591 by two red circles and the catalytic Asp by a yellow circle. In Flt3 WT, the distance between the two Tyr residues and the catalytic Asp is estimated to be 15 angstroms. In the ITD mutant, the extra stretch of residues in the JM represented by a dashed line would bridge that distance, bringing the two Tyr residues close enough for phospho-acceptance. 


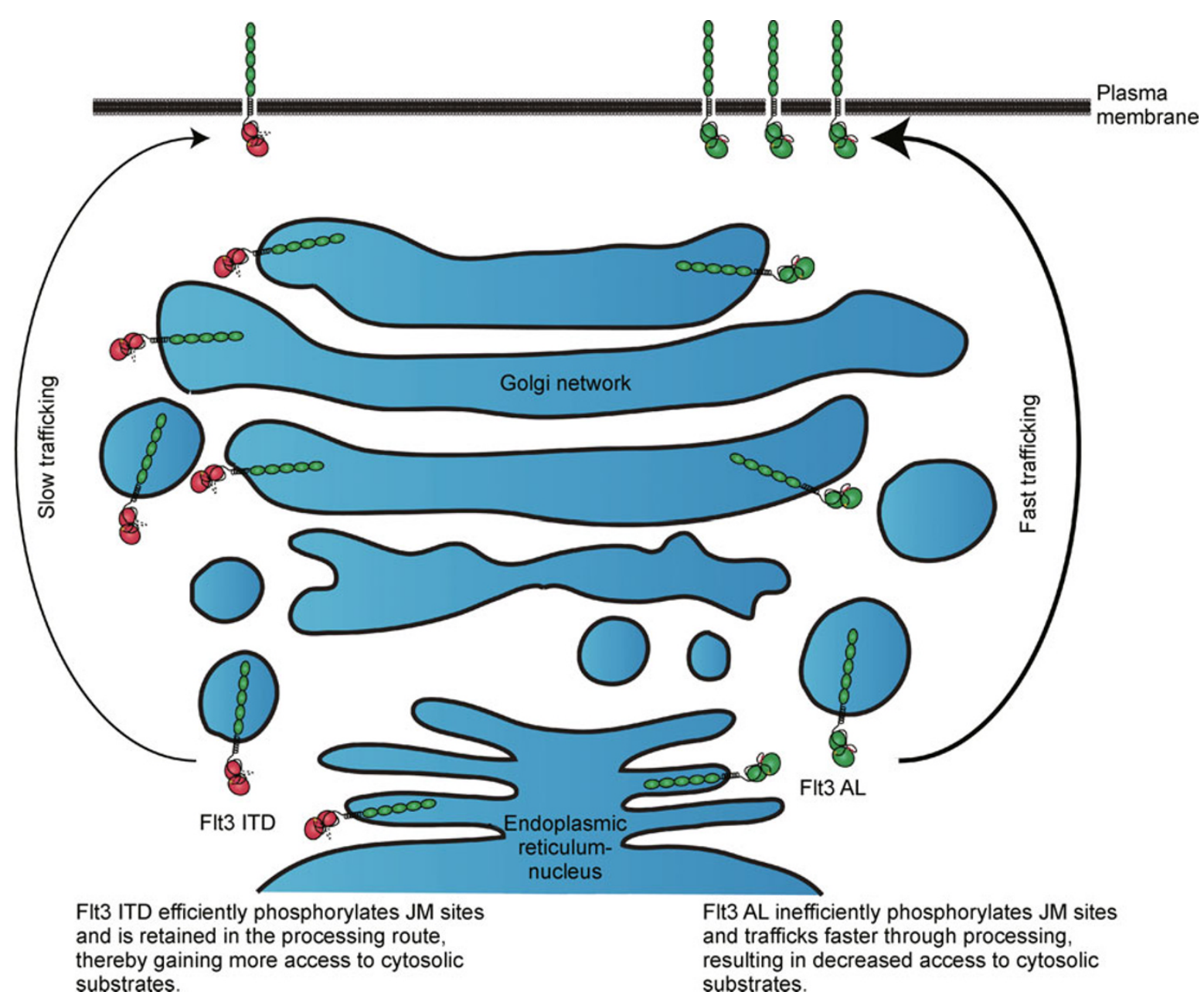

Figure 3. "Intracellular active kinase load" model. FIt3 ITD mutant autophosphorylates JM target residues early in maturation processing. The presence of phospho-JM sites either interacts directly with ER-Golgi proximal proteins or inhibits processing enzymes resident in the ER-Golgi network, resulting in retention of the receptor. The higher loads of mislocalized, activated Flt3 increase access to cytosolic substrates that are not cell surface proximal and not normally targeted by WT FIt3. FIt 3 AL mutant is less efficient in JM autophosphorylation, resulting in a lower load of mislocalized, activated kinase. Relative to FIt3 ITD, more of the AL mutant traffics through the ER-Golgi network efficiently and make the way to the plasma membrane without phosphorylation of JM sites. Flt3 AL then phosphorylates cell surface proximal substrates, like the ligand-dependent Flt3 WT signaling.

properties, then ITDs by themselves would not significantly contribute to clinical differences but rather be more like passenger mutations, although the effects of ITDs and stem cell transformation can be additive. The answer would require isolation of stage-specific cell populations using hematopoietic surface markers which are currently available. Cell genotyping by high throughput sequencing of Flt3 can then measure relative frequencies of ITD versus AL mutations, ideally at the single cell level.

Second, there is the outstanding issue of whether the Flt3 mutant kinase has switched substrate specificity through unmasking of cryptic binding sites in the JM. Identifying the interacting partners of FIt3 ITD and AL proteins by mass spectrometry of protein complexes may shed light on whether the mutant JM contains binding sites. Full-length versions of
FIt3 ITD binders can be tested as substrates to assess whether there is a preference difference.

In conclusion, therapeutic options for AML remain limited in efficacy and scope. Inhibitors against Flt3 itself have been developed almost a decade ago, but their potency remains poor (Pratz et al., 2009; Kindler et al., 2010). Establishing a mechanistic basis of Flt3 differential oncogenic signaling may provide new insights into AML therapy. Identifying novel interactors of the phosphoJM FIt3 ITD would increase the coverage of pathway components, perhaps leading to identification of other druggable targets. This is best studied in transgenic mouse inducibly expressing the Flt3 mutant (with a fluorescent tag) in hematopoietic cell lineages. Leukemic cells can be isolated by flow cytometry and analyzed by mass spectrometry for Flt3 interacting proteins 
and substrates. Of interest would be the identification of kinases and phosphatases mediating Flt3 oncogenic signaling, as more small molecule inhibitors are currently available for these two classes of enzymes.

\section{ACKNOWLEDGEMENTS}

I thank Professors W.Todd Miller (Stony Brook University, U.S.A.) and Louis Lim (University College London, UK) for helpful comments on this review.

\section{ABBREVIATIONS}

AL, activation loop; AML, acute myeloid leukemia; ERK, extracellular signal-regulated kinase; Flt3, Fms-like tyrosine kinase 3; ITDs, internal tandem duplications; JM, juxtamembrane; PTD, phosphotyrosine binding domain; RTKs, receptor tyrosine kinases; STAT5, signal transducer and activator of transcription

\section{REFERENCES}

Chan, P.M., Ilangumaran, S., La Rose, J., Chakrabartty, A., and Rottapel, R. (2003). Autoinhibition of the kit receptor tyrosine kinase by the cytosolic juxtamembrane region. Mol Cell Biol 23, 3067-3078.

Choudhary, C., Brandts, C., Schwable, J., Tickenbrock, L., Sargin, B., Ueker, A., Böhmer, F.D., Berdel, W.E., Müller-Tidow, C., and Serve, H. (2007). Activation mechanisms of STAT5 by oncogenic FIt3-ITD. Blood 110, 370-374.

Choudhary, C., Olsen, J.V., Brandts, C., Cox, J., Reddy, P.N., Böhmer, F.D., Gerke, V., Schmidt-Arras, D.E., Berdel, W.E., Müller-Tidow, C., et al. (2009). Mislocalized activation of oncogenic RTKs switches downstream signaling outcomes. Mol Cell 36, 326-339.

Choudhary, C., Schwäble, J., Brandts, C., Tickenbrock, L., Sargin, B., Kindler, T., Fischer, T., Berdel, W.E., Müller-Tidow, C., and Serve, H. (2005). AML-associated Flt3 kinase domain mutations show signal transduction differences compared with FIt3 ITD mutations. Blood 106, 265-273.

Dosil, M., Wang, S., and Lemischka, I.R. (1993). Mitogenic signalling and substrate specificity of the Flk2/FIt3 receptor tyrosine kinase in fibroblasts and interleukin 3-dependent hematopoietic cells. Mol Cell Biol 13, 6572-6585.

Gilliland, D.G., and Griffin, J.D. (2002). The roles of FLT3 in hematopoiesis and leukemia. Blood 100, 1532-1542.

Griffith, J., Black, J., Faerman, C., Swenson, L., Wynn, M., Lu, F., Lippke, J., and Saxena, K. (2004). The structural basis for autoinhibition of FLT3 by the juxtamembrane domain. Mol Cell 13, 169-178.

Grundler, R., Miething, C., Thiede, C., Peschel, C., and Duyster, J. (2005). FLT3-ITD and tyrosine kinase domain mutants induce 2 distinct phenotypes in a murine bone marrow transplantation model. Blood 105, 4792-4799.

Hayakawa, F., Towatari, M., Kiyoi, H., Tanimoto, M., Kitamura, T., Saito, H., and Naoe, T. (2000). Tandem-duplicated Flt3 constitutively activates STAT5 and MAP kinase and introduces autonomous cell growth in IL-3-dependent cell lines. Oncogene 19, 624-631.

Heiss, E., Masson, K., Sundberg, C., Pedersen, M., Sun, J.,
Bengtsson, S., and Rönnstrand, L. (2006). Identification of Y589 and Y599 in the juxtamembrane domain of Flt3 as ligand-induced autophosphorylation sites involved in binding of Src family kinases and the protein tyrosine phosphatase SHP2. Blood 108, 1542-1550.

Huntly, B.J., and Gilliland, D.G. (2005). Leukaemia stem cells and the evolution of cancer-stem-cell research. Nat Rev Cancer 5, 311-321.

Kindler, T., Lipka, D.B., and Fischer, T. (2010). FLT3 as a therapeutic target in AML: still challenging after all these years. Blood 116, 5089-5102.

Kiyoi, H., Naoe, T., Nakano, Y., Yokota, S., Minami, S., Miyawaki, S., Asou, N., Kuriyama, K., Jinnai, I., Shimazaki, C., et al. (1999). Prognostic implication of FLT3 and N-RAS gene mutations in acute myeloid leukemia. Blood 93, 3074-3080.

Kiyoi, H., Ohno, R., Ueda, R., Saito, H., and Naoe, T. (2002). Mechanism of constitutive activation of FLT3 with internal tandem duplication in the juxtamembrane domain. Oncogene 21, 2555-2563.

Koch, S., Jacobi, A., Ryser, M., Ehninger, G., and Thiede, C. (2008). Abnormal localization and accumulation of FLT3-ITD, a mutant receptor tyrosine kinase involved in leukemogenesis. Cells Tissues Organs 188, 225-235.

Kottaridis, P.D., Gale, R.E., Frew, M.E., Harrison, G., Langabeer, S. E., Belton, A.A., Walker, H., Wheatley, K., Bowen, D.T., Burnett, A. $\mathrm{K}$., et al. (2001). The presence of a FLT3 internal tandem duplication in patients with acute myeloid leukemia (AML) adds important prognostic information to cytogenetic risk group and response to the first cycle of chemotherapy: analysis of 854 patients from the United Kingdom Medical Research Council AML 10 and 12 trials. Blood 98, 1752-1759.

Levis, M., and Small, D. (2003). FLT3: ITDoes matter in leukemia. Leukemia 17, 1738-1752.

Lu, Y., Kitaura, J., Oki, T., Komeno, Y., Ozaki, K., Kiyono, M., Kumagai, H., Nakajima, H., Nosaka, T., Aburatani, H., et al. (2007). Identification of TSC-22 as a potential tumor suppressor that is upregulated by FIt3-D835V but not FIt3-ITD. Leukemia 21, 2246-2257.

Mackarehtschian, K., Hardin, J.D., Moore, K.A., Boast, S., Goff, S.P., and Lemischka, I.R. (1995). Targeted disruption of the flk2/flt3 gene leads to deficiencies in primitive hematopoietic progenitors. Immunity 3, 147-161.

Masson, K., Heiss, E., Band, H., and Rönnstrand, L. (2006). Direct binding of $\mathrm{Cbl}$ to Tyr568 and Tyr936 of the stem cell factor receptor/ c-Kit is required for ligand-induced ubiquitination, internalization and degradation. Biochem J 399, 59-67.

Masson, K., and Rönnstrand, L. (2009). Oncogenic signaling from the hematopoietic growth factor receptors c-Kit and Flt3. Cell Signal 21, 1717-1726.

Matthews, W., Jordan, C.T., Wiegand, G.W., Pardoll, D., and Lemischka, I.R. (1991). A receptor tyrosine kinase specific to hematopoietic stem and progenitor cell-enriched populations. Cell 65, 1143-1152.

Mayer, B.J., Hirai, H., and Sakai, R. (1995). Evidence that SH2 domains promote processive phosphorylation by protein-tyrosine kinases. Curr Biol 5, 296-305.

Meshinchi, S., Alonzo, T.A., Stirewalt, D.L., Zwaan, M., Zimmerman, M., Reinhardt, D., Kaspers, G.J., Heerema, N.A., Gerbing, R., 
Lange, B.J., et al. (2006). Clinical implications of FLT3 mutations in pediatric AML. Blood 108, 3654-3661.

Meshinchi, S., and Appelbaum, F.R. (2009). Structural and functional alterations of FLT3 in acute myeloid leukemia. Clin Cancer Res 15, 4263-4269.

Mol, C.D., Dougan, D.R., Schneider, T.R., Skene, R.J., Kraus, M.L., Scheibe, D.N., Snell, G.P., Zou, H., Sang, B.C., and Wilson, K.P. (2004). Structural basis for the autoinhibition and STI-571 inhibition of C-Kit tyrosine kinase. J Biol Chem 279, 31655-31663.

Mol, C.D., Lim, K.B., Sridhar, V., Zou, H., Chien, E.Y., Sang, B.C., Nowakowski, J., Kassel, D.B., Cronin, C.N., and McRee, D.E. (2003). Structure of a c-kit product complex reveals the basis for kinase transactivation. J Biol Chem 278, 31461-31464.

Murata, K., Kumagai, H., Kawashima, T., Tamitsu, K., Irie, M., Nakajima, H., Suzu, S., Shibuya, M., Kamihira, S., Nosaka, T., et al. (2003). Selective cytotoxic mechanism of GTP-14564, a novel tyrosine kinase inhibitor in leukemia cells expressing a constitutively active Fms-like tyrosine kinase 3 (FLT3). J Biol Chem 278, 32892-32898.

Nakao, M., Yokota, S., Iwai, T., Kaneko, H., Horiike, S., Kashima, K., Sonoda, Y., Fujimoto, T., and Misawa, S. (1996). Internal tandem duplication of the flt3 gene found in acute myeloid leukemia. Leukemia 10, 1911-1918.

Ong, S.E., Blagoev, B., Kratchmarova, I., Kristensen, D.B., Steen, H., Pandey, A., and Mann, M. (2002). Stable isotope labeling by amino acids in cell culture, SILAC, as a simple and accurate approach to expression proteomics. Mol Cell Proteomics 1, 376-386.

Pratz, K.W., Cortes, J., Roboz, G.J., Rao, N., Arowojolu, O., Stine, A., Shiotsu, Y., Shudo, A., Akinaga, S., Small, D., et al. (2009). A pharmacodynamic study of the FLT3 inhibitor KW-2449 yields insight into the basis for clinical response. Blood 113, 3938-3946.

Razumovskaya, E., Masson, K., Khan, R., Bengtsson, S., and Rönnstrand, L. (2009). Oncogenic Flt3 receptors display different specificity and kinetics of autophosphorylation. Exp Hematol 37, 979-989.

Rocnik, J.L., Okabe, R., Yu, J.C., Lee, B.H., Giese, N., Schenkein, D. P., and Gilliland, D.G. (2006). Roles of tyrosine 589 and 591 in STAT5 activation and transformation mediated by FLT3-ITD. Blood 108, 1339-1345.

Sargin, B., Choudhary, C., Crosetto, N., Schmidt, M.H., Grundler, R., Rensinghoff, M., Thiessen, C., Tickenbrock, L., Schwäble, J., Brandts, C., et al. (2007). Flt3-dependent transformation by inactivating c-Cbl mutations in AML. Blood 110, 1004-1012.

Schmidt-Arras, D., Böhmer, S.A., Koch, S., Müller, J.P., Blei, L., Cornils, H., Bauer, R., Korasikha, S., Thiede, C., and Böhmer, F.D.
(2009). Anchoring of FLT3 in the endoplasmic reticulum alters signaling quality. Blood 113, 3568-3576.

Small, D., Levenstein, M., Kim, E., Carow, C., Amin, S., Rockwell, P., Witte, L., Burrow, C., Ratajczak, M.Z., Gewirtz, A.M., et al. (1994). STK-1, the human homolog of Flk-2/Flt-3, is selectively expressed in CD34 + human bone marrow cells and is involved in the proliferation of early progenitor/stem cells. Proc Natl Acad Sci U S A 91, 459-463.

Stirewalt, D.L., Kopecky, K.J., Meshinchi, S., Engel, J.H., PogosovaAgadjanyan, E.L., Linsley, J., Slovak, M.L., Willman, C.L., and Radich, J.P. (2006). Size of FLT3 internal tandem duplication has prognostic significance in patients with acute myeloid leukemia. Blood 107, 3724-3726.

Stirewalt, D.L., and Radich, J.P. (2003). The role of FLT3 in haematopoietic malignancies. Nat Rev Cancer 3, 650-665.

Tickenbrock, L., Schwäble, J., Wiedehage, M., Steffen, B., Sargin, B., Choudhary, C., Brandts, C., Berdel, W.E., Müller-Tidow, C., and Serve, H. (2005). Flt3 tandem duplication mutations cooperate with Wnt signaling in leukemic signal transduction. Blood 105, 3699-3706.

Till, J.H., Chan, P.M., and Miller, W.T. (1999). Engineering the substrate specificity of the Abl tyrosine kinase. J Biol Chem 274, 4995-5003.

Vempati, S., Reindl, C., Wolf, U., Kern, R., Petropoulos, K., Naidu, V. M., Buske, C., Hiddemann, W., Kohl, T.M., and Spiekermann, K. (2008). Transformation by oncogenic mutants and ligand-dependent activation of FLT3 wild-type requires the tyrosine residues 589 and 591. Clin Cancer Res 14, 4437-4445.

Walter, M., Lucet, I.S., Patel, O., Broughton, S.E., Bamert, R., Williams, N.K., Fantino, E., Wilks, A.F., and Rossjohn, J. (2007). The $2.7 \mathrm{~A}$ crystal structure of the autoinhibited human c-Fms kinase domain. J Mol Biol 367, 839-847.

Yamamoto, Y., Kiyoi, H., Nakano, Y., Suzuki, R., Kodera, Y., Miyawaki, S., Asou, N., Kuriyama, K., Yagasaki, F., Shimazaki, C., et al. (2001). Activating mutation of D835 within the activation loop of FLT3 in human hematologic malignancies. Blood 97, 2434-2439.

Zhang, S., and Broxmeyer, H.E. (1999). p85 subunit of PI3 kinase does not bind to human Flt3 receptor, but associates with SHP2, SHIP, and a tyrosine-phosphorylated $100-\mathrm{kDa}$ protein in Flt3 ligand-stimulated hematopoietic cells. Biochem Biophys Res Commun 254, 440-445.

Zhu, H., Pan, S., Gu, S., Bradbury, E.M., and Chen, X. (2002). Amino acid residue specific stable isotope labeling for quantitative proteomics. Rapid Commun Mass Spectrom 16, 2115-2123. 